\title{
The triple collinear limit of one-loop QCD amplitudes
}

\author{
Stefano Catani ${ }^{\text {a }}$, Daniel de Florian ${ }^{\text {b }}$, Germán Rodrigo ${ }^{c, d}$

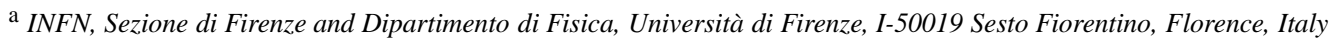 \\ ${ }^{\mathrm{b}}$ Departamento de Física, FCEYN, Universidad de Buenos Aires, (1428) Pabellón 1 Ciudad Universitaria, Capital Federal, Argentina \\ ${ }^{\mathrm{c}}$ Theory Division, CERN, CH-1211 Geneva 23, Switzerland \\ d Instituto de Física Corpuscular, E-46071 Valencia, Spain
}

Received 5 December 2003; received in revised form 28 January 2004; accepted 16 February 2004

Editor: G.F. Giudice

\begin{abstract}
We consider the singular behaviour of one-loop QCD matrix elements when several external partons become simultaneously parallel. We present a new factorization formula that describes the singular collinear behaviour directly in colour space. The collinear singularities are embodied in process-independent splitting matrices that depend on the momenta, flavours, spins and colours of the collinear partons. We give the general structure of the infrared and ultraviolet divergences of the one-loop splitting matrices. We also present explicit one-loop results for the triple collinear splitting, $q \rightarrow q \bar{Q} Q$, of a quark and a quarkantiquark pair of different flavours. The one-loop triple collinear splitting is one of the ingredients that can be used to compute the evolution of parton distributions at the next-to-next-to-leading order in QCD perturbation theory.
\end{abstract}

(c) 2004 Published by Elsevier B.V.

The high precision of experiments at past (LEP), present (HERA, Tevatron) and future (LHC, $e^{+} e^{-}$linear colliders) particle colliders demands a corresponding precision in theoretical predictions. As for perturbative QCD predictions, this means calculations beyond the next-to-leading order (NLO) in the strong coupling $\alpha_{\mathrm{S}}$. Recent years have witnessed much progress in this field (see Ref. [1] and references therein). In particular, a great deal of work has been devoted to study the properties of QCD scattering amplitudes in the infrared (soft and collinear) region [2-12].

The understanding of the infrared singular behaviour of multiparton QCD amplitudes is a prerequisite for the evaluation of infrared-finite cross sections (and, more generally, infrared- and collinear-safe QCD observables) at the next-to-next-to-leading order (NNLO) in perturbation theory [1]. The information on the infrared properties of the amplitudes have also been exploited to compute large (logarithmically enhanced) perturbative terms and to resum them to all perturbative orders [13]. The investigation of these properties is also valuable for improving the physics content of Monte Carlo event generators (see, e.g., Ref. [14]). In addition, the results of these studies prove to be useful beyond the strict QCD context, since they can provide hints on the structure of highly symmetric gauge theories at infinite orders in the perturbative expansion (see, e.g., Ref. [15]).

Work supported in part by EC 5th Framework Programme under contract number HPMF-CT-2000-00989.

E-mail addresses: stefano.catani@fi.infn.it (S. Catani), deflo@df.uba.ar (D. de Florian), german.rodrigo@cern.ch (G. Rodrigo). 
In this Letter we consider the collinear limit of multiparton QCD amplitudes at one-loop order. We present a general factorization formula, which is valid directly in colour space, and discuss some properties of its infrareddivergent contributions. These results apply to the multiple collinear limit of an arbitrary number of QCD partons. As an example of application beyond the double collinear limit, we present the result of the explicit evaluation of a triple collinear configuration of three quarks. Besides its interest within the general framework outlined above, our study of the one-loop triple collinear limit has specific relevance to the NNLO calculation of the AltarelliParisi kernels that control the scale evolution of parton densities and fragmentation functions [16]. This formidable NNLO computation is being completed [17] by using traditional methods. Kosower and Uwer [18] have proposed to exploit collinear factorization at the amplitude level as an alternative method to perform the NNLO calculation of the Altarelli-Parisi kernels. To this purpose, the one-loop triple collinear splitting is one of the necessary ingredients. Two other ingredients are the tree-level quadruple collinear splitting [11] and the two-loop double collinear splitting. A detailed discussion of the multiple collinear limit and of the results presented in this Letter will appear in a forthcoming paper [19].

We consider a generic scattering process involving final-state QCD partons (massless quarks and gluons) with momenta $p_{1}, p_{2}, \ldots$ Non-QCD partons $\left(\gamma^{*}, Z^{0}, W^{ \pm}, \ldots\right)$ are always understood. The corresponding matrix element is denoted by

$$
\mathcal{M}_{a_{1}, a_{2}, \ldots ; s_{1}, s_{2}, \ldots}^{c_{1}, c_{2}, \ldots}\left(p_{1}, p_{2}, \ldots\right),
$$

where $\left\{c_{1}, c_{2}, \ldots\right\},\left\{s_{1}, s_{2}, \ldots\right\}$ and $\left\{a_{1}, a_{2}, \ldots\right\}$, are, respectively colour, spin and flavour indices. To take into account the colour and spin structures, we use the notation of Refs. $[3,20]$. We introduce an orthonormal basis $\left\{\left|c_{1}, c_{2}, \ldots\right\rangle \otimes\left|s_{1}, s_{2}, \ldots\right\rangle\right\}$ in colour + spin space, in such a way that the matrix element in Eq. (1) can be written as

$$
\mathcal{M}_{a_{1}, a_{2}, \ldots}^{c_{1}, c_{2}, \ldots, s_{1}, s_{2}, \ldots}\left(p_{1}, p_{2}, \ldots\right) \equiv\left(\left\langle c_{1}, c_{2}, \ldots\left|\otimes\left\langle s_{1}, s_{2}, \ldots\right|\right) \mid \mathcal{M}_{a_{1}, a_{2}, \ldots}\left(p_{1}, p_{2}, \ldots\right)\right\rangle .\right.
$$

Thus $\left|\mathcal{M}_{a_{1}, a_{2}, \ldots}\left(p_{1}, p_{2}, \ldots\right)\right\rangle$ is a vector in colour + spin space. It is important to specify that the matrix elements $\mathcal{M}\left(p_{1}, p_{2}, \ldots\right)$ that we are considering are physical ones. Their external legs are on shell $\left(p_{i}^{2}=0\right)$ and have physical spin polarizations.

The matrix element $\mathcal{M}\left(p_{1}, p_{2}, \ldots\right)$ can be evaluated in QCD perturbation theory as a power series expansion (i.e., loop expansion) in the strong coupling $\alpha_{\mathrm{S}}\left(\alpha_{\mathrm{S}}=g_{\mathrm{S}}^{2} /(4 \pi)\right)$. Throughout the Letter we are mainly interested in the expansion up to one-loop order. We write

$$
\mathcal{M}=\left(g_{\mathrm{S}}\right)^{q}\left[\mathcal{M}^{(0)}+\frac{\alpha_{\mathrm{S}}}{2 \pi} \mathcal{M}^{(1)}+\mathcal{O}\left(\alpha_{\mathrm{S}}^{2}\right)\right],
$$

where the overall power $q$ is integer $(q=0,1,2,3, \ldots)$. In the evaluation of the one-loop amplitude $\mathcal{M}^{(1)}$, one encounters ultraviolet and infrared singularities that have to be properly regularized. We use dimensional regularization in $d=4-2 \epsilon$ space-time dimensions. The dimensional-regularization scale is denoted by $\mu$.

The multiple collinear limit of the matrix element in Eq. (1) is approached when the momenta $p_{1}, \ldots, p_{m}$ of $m$ partons become parallel. This implies that all the particle subenergies $s_{i j}=\left(p_{i}+p_{j}\right)^{2}$, with $i, j=1, \ldots, m$, are of the same order and vanish simultaneously. We thus introduce a pair of back-to-back light-like $\left(\tilde{P}^{2}=0, n^{2}=0\right)$ momenta $\tilde{P}^{v}$ and $n^{v}$, and we write

$$
\left(p_{1}+\cdots+p_{m}\right)^{v}=\tilde{P}^{v}+\frac{s_{1 \ldots m} n^{\nu}}{2 n \cdot \tilde{P}}, \quad s_{1 \ldots m}=\left(p_{1}+\cdots+p_{m}\right)^{2},
$$

where $s_{1 \ldots m}$ is the total invariant mass of the system of collinear partons. In the collinear limit, the vector $\tilde{P}^{v}$ denotes the collinear direction, and we have $p_{i}^{v} \rightarrow z_{i} \tilde{P}^{v}$, where the longitudinal-momentum fractions $z_{i}$ are

$$
z_{i}=\frac{n \cdot p_{i}}{n \cdot \tilde{P}}
$$


and fulfil the constraint $\sum_{i=1}^{m} z_{i}=1$. To be definite, in the rest of the Letter we limit ourselves to explicitly considering the collinear limit in the time-like region $\left(s_{i j}>0,1>z_{i}>0\right)$.

In the limit when the $m$ parton momenta $p_{1}, \ldots, p_{m}$ become simultaneously parallel, the matrix element $\mathcal{M}\left(p_{1}, \ldots, p_{m}, p_{m+1}, \ldots\right)$ becomes singular. At the tree level, the dominant singular behaviour is $\mathcal{M}^{(0)}\left(p_{1}, \ldots, p_{m}, p_{m+1}, \ldots\right) \sim(1 / \sqrt{s})^{m-1}$, where $s$ generically denotes a two-particle subenergy $s_{i j}$, or a threeparticle subenergy $s_{i j k}$, and so forth. At one-loop order, this singular behaviour is simply modified by scaling violation, $\mathcal{M}^{(1)}\left(p_{1}, \ldots, p_{m}, p_{m+1}, \ldots\right) \sim(1 / \sqrt{s})^{m-1}\left(s / \mu^{2}\right)^{-\epsilon}$. The dominant singular behaviour can be captured by universal (process-independent) factorization formulae [5-12]. At the matrix element level, the factorization formulae are usually presented upon decomposition in colour subamplitudes. Nonetheless, collinear factorization is valid directly in colour space [19], and we present the factorization formulae in this more general form.

The colour-space factorization formulae for the multiple collinear limit of the tree-level and one-loop amplitudes $\mathcal{M}^{(0)}$ and $\mathcal{M}^{(1)}$ are:

$$
\begin{aligned}
\left|\mathcal{M}_{a_{1}, \ldots, a_{m}, a_{m+1}, \ldots}^{(0)}\left(p_{1}, \ldots, p_{m}, p_{m+1}, \ldots\right)\right\rangle \simeq & \boldsymbol{S p}_{a_{1} \ldots a_{m}}^{(0)}\left(p_{1}, \ldots, p_{m}\right)\left|\mathcal{M}_{a, a_{m+1}, \ldots}^{(0)}\left(\tilde{P}, p_{m+1}, \ldots\right)\right\rangle \\
\left|\mathcal{M}_{a_{1}, \ldots, a_{m}, a_{m+1}, \ldots}^{(1)}\left(p_{1}, \ldots, p_{m}, p_{m+1}, \ldots\right)\right\rangle \simeq & \boldsymbol{S p}_{a_{1} \ldots a_{m}}^{(1)}\left(p_{1}, \ldots, p_{m}\right)\left|\mathcal{M}_{a, a_{m+1}, \ldots}^{(0)}\left(\tilde{P}, p_{m+1}, \ldots\right)\right\rangle \\
& +\boldsymbol{S p}_{a_{1} \ldots a_{m}}^{(0)}\left(p_{1}, \ldots, p_{m}\right)\left|\mathcal{M}_{a, a_{m+1}, \ldots}^{(1)}\left(\tilde{P}, p_{m+1}, \ldots\right)\right\rangle .
\end{aligned}
$$

These factorization formulae are valid in any number $d=4-2 \epsilon$ of space-time dimensions or, equivalently, at any order in the $\epsilon$ expansion around $d=4$. The only approximation involved on the right-hand side amounts to neglecting terms that are less singular in the multiple collinear limit. Eqs. (6) and (7) relate the original matrix element (on the left-hand side) with $m+k$ partons (where $k$ is arbitrary) to a matrix element (on the right-hand side) with $1+k$ partons. The latter is obtained from the former by replacing the $m$ collinear partons with a single parent parton, whose momentum is $\tilde{P}$ and whose flavour $a$ is determined by flavour conservation in the splitting process $a \rightarrow a_{1}+\cdots+a_{m}$.

The process dependence of the factorization formulae is entirely embodied in the matrix elements. The treelevel and one-loop factors $\boldsymbol{S} \boldsymbol{p}^{(0)}$ and $\boldsymbol{S \boldsymbol { p } ^ { ( 1 ) }}$, which encode the singular behaviour in the multiple collinear limit, are universal (process-independent). They depend on the momenta and quantum numbers (flavour, spin, colour) of the $m$ partons that arise from the collinear splitting. According to the notation in Eq. (2), $S p$ is a matrix in colour + spin space, and we name it the splitting matrix. Making the dependence on the colour and spin indices explicit, we have

$$
\mathrm{Sp}_{a_{1} \ldots a_{m}}^{\left(c_{1}, \ldots, c_{m} ; s_{1}, \ldots, s_{m}\right)\left(c_{a}, s_{a}\right)}=\left(\left\langlec_{1}, \ldots, c_{m}\left|\otimes\left\langle s_{1}, \ldots, s_{m}\right|\right) S \boldsymbol{p}_{a_{1} \ldots a_{m}}\left(\left|c_{a}\right\rangle \otimes\left|s_{a}\right\rangle\right),\right.\right.
$$

so that in Eqs. (6) and (7) $\boldsymbol{S p}\left(p_{1}, \ldots, p_{m}\right)$ acts onto the colour and spin indices $\left(\left\{c_{1}, \ldots, c_{m} ; s_{1}, \ldots, s_{m}\right\}\right)$ of the $m$ collinear partons on the left and onto the colour and spin indices $\left(\left\{c_{a} ; s_{a}\right\}\right)$ of the parent parton on the right.

The essential difference between Eqs. (6) and (7) and the known collinear-factorization formulae discussed in the literature $[5,9]$ regards the role of the colour quantum number. The derivation of the tree-level factorization formula (6) in colour space is quite straightforward [7]. Its one-loop extension, Eq. (7), is less straightforward and, in particular, it exploits colour coherence of QCD radiation [19]. The factorization formulae of Refs. [5,9] are written in terms of colour subamplitudes and process-independent splitting amplitudes denoted by $\operatorname{Split}\left(p_{1}, \ldots, p_{m}\right)$. Generically speaking, the splitting amplitudes Split can be regarded as colour-stripped components of the splitting matrices $\boldsymbol{S p}$. For example, in the case of the tree-level collinear splitting $g \rightarrow q_{1}+\bar{q}_{2}$, the splitting matrix is

$$
\mathrm{Sp}_{q_{1} \bar{q}_{2}}^{(0)\left(\beta_{1}, \beta_{2}\right)(c)}\left(p_{1}, p_{2}\right)=\mu^{\epsilon} t_{\beta_{1} \beta_{2}}^{c} \frac{1}{s_{12}} \bar{u}\left(p_{1}\right) \phi^{*}(\tilde{P}) v\left(p_{2}\right)
$$

where $t^{c}\left(c=1, \ldots, N_{c}^{2}-1\right)$ are the $S U\left(N_{c}\right)$ colour matrices in the fundamental representation (we use the normalization $\left.\operatorname{Tr}\left(t^{a} t^{b}\right)=T_{R} \delta^{a b}, T_{R}=1 / 2\right), u, v$ are customary Dirac spinors, $\varepsilon$ is the physical polarization vector of the parent gluon, and the corresponding spin indices are understood. The splitting amplitude Split ${ }_{q_{1}}^{(0)} \bar{q}_{2}$ is obtained from Eq. (9) by simply removing the colour factor $t_{\beta_{1} \beta_{2}}^{c}$. Analogous proportionality relations apply to any tree-level 
and one-loop splitting process $a \rightarrow a_{1}+a_{2}$. When the splitting involves $m \geqslant 3$ collinear partons, splitting matrices and splitting amplitudes are not simply proportional. Nonetheless, the two formulations of collinear factorization are related by gauge invariance and colour algebra, and each formulation has its own practical advantages.

Several general properties of the one-loop splitting amplitudes $\operatorname{Split}^{(1)}\left(p_{1}, p_{2}\right)$ and, consequently, of $\boldsymbol{S p} \boldsymbol{p}^{(1)}\left(p_{1}, p_{2}\right)$ were discussed in Refs. [5,8,10]. Here, we present an additional general property of $\boldsymbol{S} \boldsymbol{p}^{(1)}\left(p_{1}, \ldots, p_{m}\right)$.

When computed in $d=4-2 \epsilon$ space-time dimensions, the one-loop amplitude $\mathcal{M}^{(1)}$ and, hence, $\boldsymbol{S p}^{(1)}$ have ultraviolet and infrared divergences that show up as $\epsilon$-poles in the expansion around the point $\epsilon=0$. To make the divergent behaviour explicit, we write the one-loop contribution to the splitting matrix as

$$
\boldsymbol{S p}^{(1)}\left(p_{1}, \ldots, p_{m}\right)=\boldsymbol{S} \boldsymbol{p}^{(1) \operatorname{div} \cdot}\left(p_{1}, \ldots, p_{m}\right)+\boldsymbol{S p}^{(1) \mathrm{fin} .}\left(p_{1}, \ldots, p_{m}\right),
$$

where $\boldsymbol{S} \boldsymbol{p}^{(1) \text { div. }}$ behaves as $1 / \epsilon^{2}$ and $\boldsymbol{S p}^{(1) \text { fin. }}$ is finite when $\epsilon \rightarrow 0$. We also recall that different regularization schemes (RS) can actually be implemented within the dimensional-regularization prescription. Two customary RS are the conventional dimensional-regularization (CDR) scheme [21] and the dimensional-reduction (DR) scheme [22]. The RS dependence of the tree-level and one-loop splitting matrices $\boldsymbol{S} \boldsymbol{p}^{(0)}$ and $\boldsymbol{S p} \boldsymbol{p}^{(1)}$ starts at $\mathcal{O}\left(\epsilon^{0}\right)$

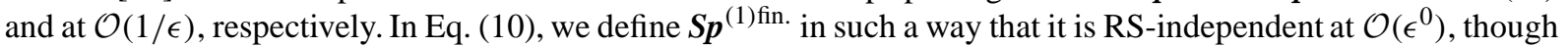
it is still RS-dependent at higher orders in the $\epsilon$-expansion. Therefore, $\boldsymbol{S} \boldsymbol{p}^{(1) \text { div. }}$ contains the full RS-dependence of $\boldsymbol{S p} \boldsymbol{p}^{(1)}$ at $\mathcal{O}\left(1 / \epsilon^{2}\right), \mathcal{O}(1 / \epsilon)$ and $\mathcal{O}\left(\epsilon^{0}\right)$.

The divergent part of the one-loop splitting matrix can be evaluated along the lines of Refs. [3,20,24], and the result can be expressed in terms of a process-independent factorization formula. We obtain [19]

$$
\begin{aligned}
& \boldsymbol{S p}^{(1) \text { div. }\left(p_{1}, \ldots, p_{m}\right)} \\
& \begin{aligned}
&=\frac{\Gamma(1+\epsilon) \Gamma^{2}(1-\epsilon)}{(4 \pi)^{-\epsilon} \Gamma(1-2 \epsilon)} \frac{1}{2}\left\{\frac{1}{\epsilon^{2}} \sum_{i, j=1(i \neq j)}^{m} \boldsymbol{T}_{i} \cdot \boldsymbol{T}_{j}\left(\frac{-s_{i j}-i 0}{\mu^{2}}\right)^{-\epsilon}\right. \\
&+\left(\frac{-s_{1 \ldots m}-i 0}{\mu^{2}}\right)^{-\epsilon}\left[\frac{1}{\epsilon^{2}} \sum_{i, j=1}^{m} \boldsymbol{T}_{i} \cdot \boldsymbol{T}_{j}\left(2-\left(z_{i}\right)^{-\epsilon}-\left(z_{j}\right)^{-\epsilon}\right)\right. \\
&\left.\left.\quad-\frac{1}{\epsilon}\left(\sum_{i=1}^{m}\left(\gamma_{i}-\epsilon \tilde{\gamma}_{i}^{\mathrm{RS}}\right)-\left(\gamma_{a}-\epsilon \tilde{\gamma}_{a}^{\mathrm{RS}}\right)-\frac{m-1}{2}\left(\beta_{0}-\epsilon \tilde{\beta}_{0}^{\mathrm{RS}}\right)\right)\right]\right\} \\
& \times \boldsymbol{S p}^{(0)}\left(p_{1}, \ldots, p_{m}\right),
\end{aligned}
\end{aligned}
$$

where we have used the same notation as in Ref. [3]. The colour charge (matrix) of the collinear parton with momentum $p_{i}$ is denoted by $\boldsymbol{T}_{i}$, and colour conservation implies $\sum_{i} \boldsymbol{T}_{i} \boldsymbol{S} \boldsymbol{p}^{(0)}=\boldsymbol{S} \boldsymbol{p}^{(0)} \boldsymbol{T}_{a}\left(\boldsymbol{T}_{a}\right.$ is the colour charge of the parent parton in the collinear splitting). The flavour coefficients $\gamma_{i}$ and $\beta_{0}$ are $\gamma_{q}=\gamma_{\bar{q}}=3 C_{F} / 2$ and $\gamma_{g}=\beta_{0} / 2=\left(11 C_{A}-2 N_{f}\right) / 6$. The flavour coefficients $\tilde{\gamma}_{i}^{\mathrm{RS}}$ and $\tilde{\beta}_{0}^{\mathrm{RS}}$ are RS-dependent [23]. In particular, $\tilde{\gamma}_{i}^{\mathrm{CDR}}=\tilde{\beta}_{0}^{\mathrm{CDR}}=0$, while $\tilde{\gamma}_{q}^{\mathrm{DR}}=\tilde{\gamma}_{\bar{q}}^{\mathrm{DR}}=C_{F} / 2$ and $\tilde{\gamma}_{g}^{\mathrm{DR}}=\tilde{\beta}_{0}^{\mathrm{DR}} / 2=C_{A} / 6$.

Note that, so far, we have not specified whether the one-loop amplitudes $\mathcal{M}^{(1)}$ and splitting matrices $\boldsymbol{S p}^{(1)}$ are renormalized or unrenormalized quantities. Since the renormalization procedure commutes with the collinear limit, the factorization formula (7) equally applies to both renormalized and unrenormalized quantities. However, the divergent part $\boldsymbol{S p}^{(1) \text { div. }}$ explicitly given in Eq. (11) refers to the (charge) unrenormalized splitting matrix (thus, $\alpha_{\mathrm{S}}$ is the bare QCD coupling). In the curly bracket of Eq. (11), the contribution proportional to $\beta_{0}-\epsilon \tilde{\beta}_{0}^{\mathrm{RS}}$ is of ultraviolet origin, and it would disappear by working at the level of renormalized matrix elements and splitting matrices.

It is straightforward to check that Eq. (11) agrees with the divergent behaviour of the known $[5,8,10]$ one-loop splitting amplitudes for the double collinear limit $a \rightarrow a_{1}+a_{2}$. The triple collinear splitting process explicitly considered below provides us with a further check of the general result in Eq. (11). 
At the tree level, the splitting amplitudes are known for all possible partonic channels in the double $[25,26]$ and triple [11] collinear limits, and for pure gluonic splitting $(g \rightarrow 4 g)$ also in the quadruple collinear case [11]. In the double collinear case, the square of the splitting amplitudes gives the leading-order Altarelli-Parisi kernels [27]. The square of the triple collinear splitting amplitudes was computed in Refs. [6,7], and checked in Ref. [11].

At the one-loop level, the splitting amplitudes for all partonic channels in the double collinear limit were computed in Refs. $[8,10]$ to all orders in $\epsilon$. In the following we consider the triple collinear limit and, more precisely, the collinear splitting process $q \rightarrow q_{1}+\bar{Q}_{2}+Q_{3}$, where $q$ and $Q$ denote (massless) quarks of different flavours.

We first recall the tree-level splitting matrix for the process $q \rightarrow q\left(p_{1}\right)+\bar{Q}\left(p_{2}\right)+Q\left(p_{3}\right)$ :

$$
\operatorname{Sp}_{q_{1} \bar{Q}_{2} Q_{3}}^{(0)\left(\beta_{1}, \beta_{2}, \beta_{3}\right)(\beta)}\left(p_{1}, p_{2}, p_{3}\right)=\mu^{2 \epsilon} \sum_{c} t_{\beta_{3} \beta_{2}}^{c} t_{\beta_{1} \beta}^{c} \frac{1}{s_{123} S_{23}} \bar{u}\left(p_{3}\right) \gamma^{\mu} v\left(p_{2}\right) \bar{u}\left(p_{1}\right) \gamma^{v} u(\tilde{P}) d_{\mu v}\left(p_{2}+p_{3}, n\right),
$$

where $\beta_{i}(i=1,2,3)$ and $\beta$ are respectively the colour indices of the final-state fermions and of the parent quark, and

$$
d^{\mu \nu}(k, n)=-g^{\mu \nu}+\frac{k^{\mu} n^{v}+n^{\mu} k^{\nu}}{n \cdot k}
$$

is the physical polarization tensor of the gluon with momentum $k$ ( $n^{\mu}$ is the auxiliary light-like vector introduced in Eq. (4)).

The square of the splitting matrix $\boldsymbol{S} \boldsymbol{p}_{a_{1} \ldots a_{m}}$, summed over final-state colours and spins and averaged over colours and spins of the parent parton, defines the $m$-parton splitting function $\left\langle\hat{P}_{a_{1} \ldots a_{m}}\right\rangle$, which is a generalization of the customary (i.e., with $m=2$ ) Altarelli-Parisi splitting function. Fixing the normalization of the tree-level splitting function $\left\langle\hat{P}_{a_{1} \ldots a_{m}}^{(0)}\right\rangle$ by

$$
\left\langle\hat{P}_{a_{1} \ldots a_{m}}^{(0)}\right\rangle=\left(\frac{s_{1 \ldots m}}{2 \mu^{2 \epsilon}}\right)^{m-1} \overline{\left|\boldsymbol{S p}_{a_{1} \ldots a_{m}}^{(0)}\right|^{2}}
$$

from Eq. (12) we have

$$
\left\langle\hat{P}_{q_{1} \bar{Q}_{2} Q_{3}}^{(0)}\right\rangle=\frac{1}{2} C_{F} T_{R} \frac{s_{123}}{s_{23}}\left[-\frac{t_{23,1}^{2}}{s_{23} s_{123}}+\frac{4 z_{1}+\left(z_{2}-z_{3}\right)^{2}}{z_{2}+z_{3}}+\left(1-2 \epsilon \delta^{\mathrm{RS}}\right)\left(z_{2}+z_{3}-\frac{s_{23}}{s_{123}}\right)\right],
$$

where

$$
t_{i j, k} \equiv 2 \frac{z_{i} s_{j k}-z_{j} s_{i k}}{z_{i}+z_{j}}+\frac{z_{i}-z_{j}}{z_{i}+z_{j}} s_{i j}
$$

which agrees with the result obtained in Refs. [6,7]. The parameter $\delta^{\mathrm{RS}}$ depends on the RS: $\delta^{\mathrm{CDR}}=1$ and $\delta^{\mathrm{DR}}=0$.

To evaluate the one-loop splitting matrix $\boldsymbol{S p}_{a_{1} \ldots a_{m}}^{(1)}$ we use a process-independent method $[7,9,19]$. In the case of the collinear splitting process $q \rightarrow q_{1}+\bar{Q}_{2}+Q_{3}, S p_{q_{1} \bar{Q}_{2} Q_{3}}^{(1)}$ receives a contribution from two different colour structures:

$$
\begin{aligned}
\operatorname{Sp}_{q_{1} \bar{Q}_{2} Q_{3}}^{(1)\left(\beta_{1}, \beta_{2}, \beta_{3}\right)(\beta)}\left(p_{1}, p_{2}, p_{3}\right)=\mu^{4 \epsilon} \frac{8 \pi^{2}}{s_{123}} & \left\{\sum_{c} t_{\beta_{3} \beta_{2}}^{c} t_{\beta_{1} \beta}^{c} \mathcal{S}\left(p_{1}, p_{2}, p_{3}\right)\right. \\
& \left.+\sum_{b, c}\left(t^{b} t^{c}+t^{c} t^{b}\right)_{\beta_{3} \beta_{2}}\left(t^{c} t^{b}\right)_{\beta_{1} \beta} \mathcal{A}\left(p_{1}, p_{2}, p_{3}\right)\right\} .
\end{aligned}
$$

The first term in the curly bracket has the same structure as the tree-level contribution in Eq. (12). The colour structure of the second term is a new one-loop (quantum) effect.

The one-loop correction $\hat{P}^{(1)}$ to the tree-level splitting function is obtained by simply performing the replacement $\left|\boldsymbol{S} \boldsymbol{p}^{(0)}\right|^{2} \rightarrow\left(\boldsymbol{S} \boldsymbol{p}^{(0)}\right)^{\dagger} \boldsymbol{S} \boldsymbol{p}^{(1)}+\left(\boldsymbol{S} \boldsymbol{p}^{(1)}\right)^{\dagger} \boldsymbol{S} \boldsymbol{p}^{(0)}$ on the right-hand side of Eq. (14). From Eqs. (12) and (17), 


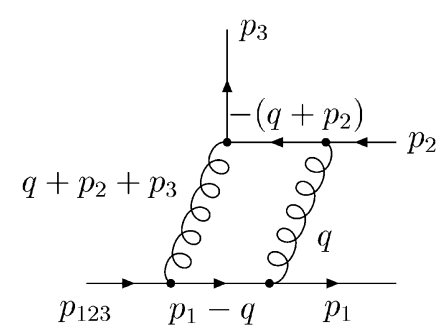

(a)

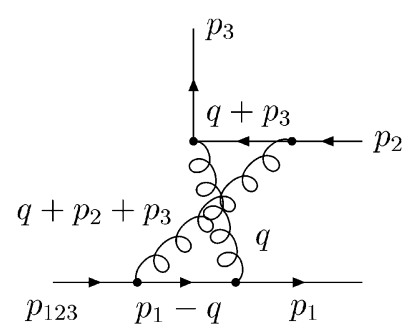

(b)

Fig. 1. One-loop diagrams contributing to the antisymmetric component of the splitting matrix $\boldsymbol{S p}{ }_{q_{1}} \bar{Q}_{2} Q_{3}$.

we obtain

$$
\left\langle\hat{P}_{q_{1} \bar{Q}_{2} Q_{3}}\right\rangle=\left\langle\hat{P}_{q_{1} \bar{Q}_{2} Q_{3}}^{(0)}\right\rangle+\frac{\alpha_{\mathrm{S}}}{2 \pi}\left[\left\langle\hat{P}_{q_{1} \bar{Q}_{2} Q_{3}}^{(1)(\text { sym. })}\right\rangle+\left\langle\hat{P}_{q_{1} \bar{Q}_{2} Q_{3}}^{(1)(\text { an })}\right\rangle\right]+\mathcal{O}\left(\alpha_{\mathrm{S}}^{2}\right) .
$$

Note that $\left\langle\hat{P}_{q_{1} \bar{Q}_{2} Q_{3}}^{(0)}\right\rangle$ is symmetric with respect to the exchange $p_{2} \leftrightarrow p_{3}$ of the $\bar{Q}_{2}$ and $Q_{3}$ momenta (see Eq. (15)). Its one-loop correction has, instead, a symmetric component and an antisymmetric component, respectively denoted by $\left\langle\hat{P}_{q_{1} \bar{Q}_{2} Q_{3}}^{(1)(\text { sym. }}\right\rangle$ and $\left\langle\hat{P}_{q_{1} \bar{Q}_{2} Q_{3}}^{(1)(\text { an.) }}\right\rangle$. The antisymmetric component is entirely produced by the one-loop splitting amplitude $\mathcal{A}\left(p_{1}, p_{2}, p_{3}\right)$ on the right-hand side of Eq. (17).

The expression of the splitting amplitude $\mathcal{A}\left(p_{1}, p_{2}, p_{3}\right)$ is sufficiently compact to be presented explicitly in this Letter. We have

$$
\begin{aligned}
\mathcal{A}\left(p_{1}, p_{2}, p_{3}\right)= & -\frac{1}{2} i \int \frac{d^{d} q}{(2 \pi)^{d}} \bar{u}\left(p_{3}\right)\left[\frac{\gamma^{\sigma}\left(\not d+\not p_{2}\right) \gamma^{\mu}}{\left(s_{2 q}+i 0\right)}-\frac{\gamma^{\mu}\left(\not d+\not p_{3}\right) \gamma^{\sigma}}{\left(s_{3 q}+i 0\right)}\right] v\left(p_{2}\right) \\
& \times d_{\mu \nu}(q, n) d_{\sigma \rho}\left(q+p_{2}+p_{3}, n\right) \frac{\bar{u}\left(p_{1}\right) \gamma^{\nu}\left(p_{1}-\not q\right) \gamma^{\rho} u(\tilde{P})}{\left(q^{2}+i 0\right)\left(t_{1 q}+i 0\right)\left(s_{23 q}+i 0\right)},
\end{aligned}
$$

where

$$
t_{1 q}=\left(p_{1}-q\right)^{2}, \quad s_{i q}=\left(p_{i}+q\right)^{2}, \quad s_{23 q}=\left(p_{2}+p_{3}+q\right)^{2} .
$$

The first and second contributions in the square bracket originate from the one-loop diagrams depicted in Fig. 1(a) and (b), respectively.

Note that the expression in Eq. (19) is valid in any RS. It is also valid in any number $d=4-2 \epsilon$ of space-time dimensions or, equivalently, to all orders in $\epsilon$. To make the dependence on $\epsilon$ explicit, we have to compute the $d$-dimensional integral over the loop momentum $q$. This computation requires the evaluation of a set of basic one-loop (scalar and tensor) integrals. The corresponding integrands involve, besides the customary Feynman propagators $1 /\left(q^{2}+i 0\right)$, additional propagators of the type $1 /(n \cdot q)$, which come from the physical polarizations of the virtual gluons (see Eq. (13)). Some of these integrals, which resemble those encountered in axial-gauge calculations, were evaluated by Kosower and Uwer [10] in the context of their calculation of the one-loop double collinear splitting $a \rightarrow a_{1}+a_{2}$. More complicated integrals (higher-point functions) of this type are involved in triple collinear splitting processes. We have computed (to high orders in the $\epsilon$ expansion) all the basic one-loop integrals [19] that appear in any triple collinear splitting $a \rightarrow a_{1}+a_{2}+a_{3}$. Using these results, we have obtained explicit expressions up to $\mathcal{O}\left(\epsilon^{0}\right)$ of the splitting amplitude $\mathcal{A}\left(p_{1}, p_{2}, p_{3}\right)$ in Eqs. (17), (19) and of the corresponding splitting function $\left\langle\hat{P}_{q_{1} \bar{Q}_{2} Q_{3}}^{(1)(\text { an.) }}\right\rangle$ in Eq. (18). We limit ourselves to presenting the expression of $\left\langle\hat{P}_{q_{1} \bar{Q}_{2} Q_{3}}^{(1)(\text { an })}\right\rangle$, since the expression of $\mathcal{A}\left(p_{1}, p_{2}, p_{3}\right)$ has a very similar structure. 
Including terms up to $\mathcal{O}\left(\epsilon^{0}\right)$, our result for the antisymmetric component of the one-loop splitting function is

$$
\begin{aligned}
\left\langle\hat{P}_{q_{1} \bar{Q}_{2} Q_{3}}^{(1) \text { (an. })}\right\rangle= & \frac{\Gamma(1+\epsilon) \Gamma^{2}(1-\epsilon)}{(4 \pi)^{-\epsilon} \Gamma(1-2 \epsilon)} C_{F} T_{R} \frac{N_{c}^{2}-4}{4 N_{c}}\left(\frac{-s_{123}-i 0}{\mu^{2}}\right)^{-\epsilon} \\
& \times\left(\left\{\frac { 1 } { \epsilon ^ { 2 } } \left[\left(\left(\frac{s_{23}}{s_{123}}\right)^{-\epsilon}+1\right)\left(\left(\frac{z_{2}}{z_{2}+z_{3}}\right)^{-\epsilon}-\left(\frac{z_{3}}{z_{2}+z_{3}}\right)^{-\epsilon}+\left(\frac{s_{13}}{s_{123}}\right)^{-\epsilon}-\left(\frac{s_{12}}{s_{123}}\right)^{-\epsilon}\right)\right.\right.\right. \\
& \left.\left.+\left(\frac{s_{12}}{s_{123}}\right)^{-\epsilon}\left(\frac{z_{3}}{z_{2}+z_{3}}\right)^{-\epsilon}-\left(\frac{s_{13}}{s_{123}}\right)^{-\epsilon}\left(\frac{z_{2}}{z_{2}+z_{3}}\right)^{-\epsilon}\right]\right\}\left\langle\hat{P}_{q_{1} \bar{Q}_{2} Q_{3}}^{(0)}\right\rangle /\left(C_{F} T_{R}\right) \\
+ & \left\{\left(\hat{a}-\frac{s_{13}}{s_{123}-s_{13}} \hat{b}\right) \ln \left(\frac{s_{13}}{s_{123}}\right)+\left(\frac{s_{23}}{s_{123}-s_{23}} \hat{a}-\hat{b}\right) \ln \left(\frac{s_{23}}{s_{123}}\right)\right. \\
& +\frac{s_{123} s_{12}}{s_{12}+s_{13}-z_{1} s_{123}}\left(\frac{z_{2} \hat{a}-z_{1} \hat{b}}{s_{12}\left(1-z_{1}\right)}+\frac{\hat{a}+\hat{b}}{s_{123}}\right) \ln \left(\frac{s_{12} z_{2}}{s_{13} z_{3}}\right) \ln \left(\frac{s_{23}}{s_{123}\left(z_{2}+z_{3}\right)}\right) \\
& +\frac{\left(2 s_{12}+s_{23}\right) \hat{a}+\left(s_{12}-s_{13}\right) \hat{b}}{s_{12}} \\
& \left.\left.\quad \times\left[\ln \left(\frac{s_{13}}{s_{123}}\right) \ln \left(\frac{s_{23}}{s_{123}}\right)+\operatorname{Li}_{2}\left(1-\frac{s_{13}}{s_{123}}\right)+\operatorname{Li}_{2}\left(1-\frac{s_{23}}{s_{123}}\right)-\frac{\pi^{2}}{6}\right]-(2 \leftrightarrow 3)\right\}+\mathcal{O}(\epsilon)\right) \\
+ & \text { complex conjugate, }
\end{aligned}
$$

where

$$
\begin{aligned}
& \hat{a}=\frac{s_{123}}{s_{23}}\left(\frac{z_{1} s_{13}}{s_{123}}+\frac{z_{1}\left(z_{1} s_{23}-z_{2} s_{13}-z_{3} s_{12}\right)}{2\left(z_{2}+z_{3}\right) s_{12}}+\frac{z_{1} s_{23}+z_{2} s_{13}-z_{3} s_{12}}{2 s_{123}}\right), \\
& \hat{b}=\frac{s_{123}}{s_{23}}\left(\frac{z_{2} s_{23}}{s_{123}}+\frac{z_{2}\left(z_{1} s_{23}-z_{2} s_{13}+z_{3} s_{12}\right)}{2\left(z_{2}+z_{3}\right) s_{12}}+\frac{z_{1} s_{23}+z_{2} s_{13}-z_{3} s_{12}}{2 s_{123}}\right) .
\end{aligned}
$$

It is quite straightforward to check that the divergent part of the one-loop splitting function in Eq. (21) fully agrees with the result obtained by using the general formula in Eq. (11). Note that the double poles $1 / \epsilon^{2}$ cancel in Eq. (21), so that the most divergent terms in $\left\langle\hat{P}_{q_{1}}^{(1)\left(\bar{Q}_{2} Q_{3}\right)}\right\rangle$ are single poles $1 / \epsilon$. These single poles originate from the infrared region of the loop integral in Eq. (19). More precisely, they arise when the loop momentum $q$ is soft $(q \rightarrow 0)$ but not collinear to any of the external momenta. Note also that, up to terms of $\mathcal{O}\left(\epsilon^{0}\right)$, the full RS dependence of $\left\langle\hat{P}_{q_{1} \bar{Q}_{2} Q_{3}}^{(1) \text { (an.) }}\right\rangle$ is embodied in the corresponding dependence (see Eq. (15)) of the tree-level term $\left\langle\hat{P}_{q_{1} \bar{Q}_{2} Q_{3}}^{(0)}\right\rangle$ on the right-hand side of Eq. (21). The absence of additional RS-dependent terms (such as those proportional to $\tilde{\gamma}_{i}^{\mathrm{RS}}$ and $\tilde{\beta}_{0}^{\mathrm{RS}}$ in Eq. (11)) at $\mathcal{O}\left(\epsilon^{0}\right)$ is related to the absence of single poles $1 / \epsilon$ of ultraviolet and collinear origin, as discussed in the second paper of Ref. [23].

An additional check of our result can be performed by considering the triple collinear limit of known one-loop matrix elements, such as the matrix element for the process $e^{+} e^{-} \rightarrow \bar{q} q \bar{Q} Q[28,29]$. We have evaluated the oneloop splitting amplitude (19) in a spin basis of definite \pm 1 helicities of the quarks and antiquarks. We have then compared the result with that obtained by directly performing the corresponding collinear limit of the one-loop helicity amplitudes explicitly presented in Ref. [28] up to $\mathcal{O}\left(\epsilon^{0}\right)$ (note that the expressions in Ref. [28] refer to the DR scheme). We have found complete agreement.

The splitting function $\hat{P}_{a_{1} \ldots a_{m}}$ controls the singular behaviour in the multiple collinear limit. Moreover, it can have additional singularities (i.e., terms that are not integrable in $d=4$ dimensions) in some subregions of the collinear phase-space. The tree-level splitting function $\left\langle\hat{P}_{q_{1} \bar{Q}_{2} Q_{3}}^{(0)}\right\rangle$ in Eq. (15) is indeed singular when the momenta $p_{2}$ and $p_{3}$ are parallel (i.e., when their relative angle is much smaller than the emission angle of $p_{1}$ ) and when they are simultaneously soft. The singularity when $p_{2}$ and $p_{3}$ are parallel is, instead, absent (to any order in the 
$\epsilon$ expansion [19], and not only at $\left.\mathcal{O}\left(\epsilon^{0}\right)\right)$ in the antisymmetric part, $\left\langle\hat{P}_{q_{1}}^{(1)\left(\bar{Q}_{2} Q_{3}\right.}\right\rangle$, of the one-loop splitting function. The singularity when $p_{2}$ and $p_{3}$ are simultaneously soft is still present in $\left\langle\hat{P}_{q_{1} \bar{Q}_{2} Q_{3}}^{(1) \text { (an.) }}\right\rangle$, though its effect vanishes after integration over the angles of $p_{2}$ and $p_{3}$ because of the antisymmetry with respect to the exchange $p_{2} \leftrightarrow p_{3}$. The expression in Eq. (21) shows that $\left\langle\hat{P}_{q_{1} \bar{Q}_{2} Q_{3}}^{(1)(\text { an. }}\right\rangle$ has no other singularities in any subregions of the phase-space.

For the sake of brevity, we have limited ourselves, in this Letter, to presenting a few explicit results for the one-loop triple collinear splitting. These results have mainly an illustrative purpose. The method and the tools (in particular, the one-loop integrals) used to obtain them are sufficient and can be applied straightforwardly to evaluate the one-loop splitting matrix of any splitting process $a \rightarrow a_{1}+a_{2}+a_{3}$.

\section{Acknowledgements}

We wish to thank Werner Vogelsang for collaboration at an early stage of this work. D.d.F. thanks Fundación Antorchas and Conicet for partial support. G.R. thanks Generalitat Valenciana under grants CTIDIB/2002/24 and GRUPOS03/013, and MCyT under grant FPA-2001-3031 for partial support.

\section{References}

[1] T. Gehrmann, Nucl. Phys. B (Proc. Suppl.) 116 (2003) 13, hep-ph/0210157; E.W. Glover, Nucl. Phys. B (Proc. Suppl.) 116 (2003) 3, hep-ph/0211412; Z. Bern, Nucl. Phys. B (Proc. Suppl.) 117 (2003) 260, hep-ph/0212406.

[2] F.A. Berends, W.T. Giele, Nucl. Phys. B 313 (1989) 595.

[3] S. Catani, Phys. Lett. B 427 (1998) 161, hep-ph/9802439.

[4] S. Catani, M. Grazzini, Nucl. Phys. B 591 (2000) 435, hep-ph/0007142.

[5] Z. Bern, L.J. Dixon, D.C. Dunbar, D.A. Kosower, Nucl. Phys. B 425 (1994) 217, hep-ph/9403226.

[6] J.M. Campbell, E.W. Glover, Nucl. Phys. B 527 (1998) 264, hep-ph/9710255.

[7] S. Catani, M. Grazzini, Phys. Lett. B 446 (1999) 143, hep-ph/9810389; S. Catani, M. Grazzini, Nucl. Phys. B 570 (2000) 287, hep-ph/9908523.

[8] Z. Bern, V. Del Duca, C.R. Schmidt, Phys. Lett. B 445 (1998) 168, hep-ph/9810409; Z. Bern, V. Del Duca, W.B. Kilgore, C.R. Schmidt, Phys. Rev. D 60 (1999) 116001, hep-ph/9903516.

[9] D.A. Kosower, Nucl. Phys. B 552 (1999) 319, hep-ph/9901201.

[10] D.A. Kosower, P. Uwer, Nucl. Phys. B 563 (1999) 477, hep-ph/9903515.

[11] V. Del Duca, A. Frizzo, F. Maltoni, Nucl. Phys. B 568 (2000) 211, hep-ph/9909464.

[12] D.A. Kosower, Phys. Rev. D 67 (2003) 116003, hep-ph/0212097;

D.A. Kosower, Phys. Rev. Lett. 91 (2003) 061602, hep-ph/0301069;

D.A. Kosower, preprint SACLAY-SPHT-T03-178, hep-ph/0311272.

[13] D. de Florian, M. Grazzini, Phys. Rev. Lett. 85 (2000) 4678, hep-ph/0008152;

D. de Florian, M. Grazzini, Nucl. Phys. B 616 (2001) 247, hep-ph/0108273;

S. Catani, D. de Florian, M. Grazzini, JHEP 0105 (2001) 025, hep-ph/0102227;

S. Catani, D. de Florian, M. Grazzini, P. Nason, JHEP 0307 (2003) 028, hep-ph/0306211.

[14] CERN Workshop on Monte Carlo tools for the LHC, CERN, Geneva, July 2003, see: http://mlm.home.cern.ch/mlm/mcwshop03/ mcwshop.html.

[15] C. Anastasiou, Z. Bern, L.J. Dixon, D.A. Kosower, Phys. Rev. Lett. 91 (2003) 251602, hep-th/0309040.

[16] R.K. Ellis, W.J. Stirling, B.R. Webber, QCD and Collider Physics, Cambridge Univ. Press, Cambridge, 1996, and references therein.

[17] S.A. Larin, P. Nogueira, T. van Ritbergen, J.A. Vermaseren, Nucl. Phys. B 492 (1997) 338, hep-ph/9605317;

A. Retey, J.A. Vermaseren, Nucl. Phys. B 604 (2001) 281, hep-ph/0007294;

S. Moch, J.A. Vermaseren, A. Vogt, Nucl. Phys. B 646 (2002) 181, hep-ph/0209100;

S. Moch, J.A. Vermaseren, A. Vogt, Nucl. Phys. B (Proc. Suppl.) 116 (2003) 100, hep-ph/0211296.

[18] D.A. Kosower, P. Uwer, Nucl. Phys. B 674 (2003) 365, hep-ph/0307031.

[19] S. Catani, D. de Florian, G. Rodrigo, in preparation.

[20] S. Catani, M.H. Seymour, Nucl. Phys. B 485 (1997) 291;

S. Catani, M.H. Seymour, Nucl. Phys. B 510 (1997) 503, Erratum, hep-ph/9605323. 
[21] G. 't Hooft, M.J. Veltman, Nucl. Phys. B 44 (1972) 189;

C.G. Bollini, J.J. Giambiagi, Nuovo Cimento B 12 (1972) 20;

J.F. Ashmore, Lett. Nuovo Cimento 4 (1972) 289;

G.M. Cicuta, E. Montaldi, Lett. Nuovo Cimento 4 (1972) 329;

R. Gastmans, R. Meuldermans, Nucl. Phys. B 63 (1973) 277.

[22] W. Siegel, Phys. Lett. B 84 (1979) 193;

Z. Bern, D.A. Kosower, Nucl. Phys. B 379 (1992) 451;

Z. Bern, A. De Freitas, L.J. Dixon, H.L. Wong, Phys. Rev. D 66 (2002) 085002, hep-ph/0202271.

[23] Z. Kunszt, A. Signer, Z. Trocsanyi, Nucl. Phys. B 411 (1994) 397, hep-ph/9305239;

S. Catani, M.H. Seymour, Z. Trocsanyi, Phys. Rev. D 55 (1997) 6819, hep-ph/9610553.

[24] S. Catani, S. Dittmaier, Z. Trocsanyi, Phys. Lett. B 500 (2001) 149, hep-ph/0011222.

[25] F.A. Berends, W.T. Giele, Nucl. Phys. B 306 (1988) 759.

[26] M.L. Mangano, S.J. Parke, Phys. Rep. 200 (1991) 301.

[27] G. Altarelli, G. Parisi, Nucl. Phys. B 126 (1977) 298.

[28] Z. Bern, L.J. Dixon, D.A. Kosower, S. Weinzierl, Nucl. Phys. B 489 (1997) 3, hep-ph/9610370;

Z. Bern, L.J. Dixon, D.A. Kosower, Nucl. Phys. B 513 (1998) 3, hep-ph/9708239.

[29] E.W.N. Glover, D.J. Miller, Phys. Lett. B 396 (1997) 257, hep-ph/9609474. 